\title{
Contribuciones de la biblioteca universitaria a la internacionalización del campus: análisis sistemático de la literatura
}

Nancy Sánchez-Tarragó

\begin{abstract}
Doutora em Informação e Documentação, Universidad de Granada, España. Professora Facultad de Comunicación, Universidad de La Habana, Cuba, Estagiária de Pós-doutorado no Departamento de Ciência da Informação. Universidade Federal de Pernambuco
\end{abstract}

Inalvis Castellanos-Gallardo,

Master en Ciencia de la Información. Universidad de La Habana/ Equador

Leilah Santiago Bufrem

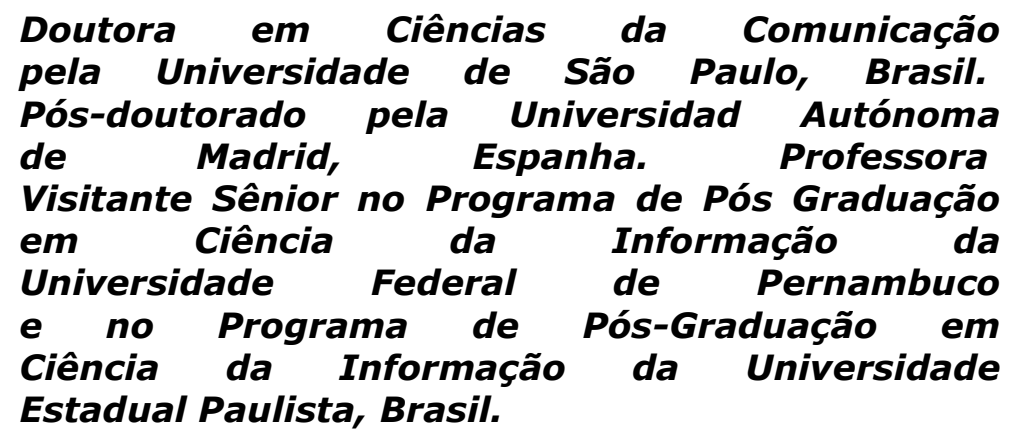

http://dx.doi.org/10.1590/1981-5344/2968

Los procesos de internacionalización universitaria constituyen respuesta adaptativa a un contexto mundial signado por la globalización, que implica, grosso modo, mayor interrelación política, económica y social entre países y regiones. En este contexto, es válido preguntarse cuál es el papel y las contribuciones de la biblioteca universitaria en los procesos de internacionalización del campus, teniendo en cuenta que, históricamente, su razón de ser ha sido servir de apoyo a la universidad en el desarrollo de sus funciones. Para encontrar respuestas se realizó una revisión sistemática de la literatura publicada en Ciencia de la información entre el 2005 y el 2015 y un análisis bibliométrico de esa producción científica. Se constató un crecimiento continuo de la investigación publicada, liderada por bibliotecarios de Estados Unidos, 
China y Canadá, que abordan diferentes aristas del trabajo bibliotecario que apoyan tanto la educación transnacional como la internacionalización 'en casa': servicios bibliotecarios para apoyar estudiantes y programas en el exterior, programas de alfabetización informacional, estudios sobre la influencia de las diferencias culturales y lingüísticas en la comunicación y los servicios, desarrollo de colecciones, entre otras. Se identificaron recomendaciones para la práctica bibliotecaria relacionadas con estrategias administrativas, de servicios, infraestructura, formación e investigación.

Palavras-chave: Internacionalización; Educación Superior; Bibliotecas Universitarias; Prácticas Bibliotecarias.

\section{Academic library contributions to internationalization: a systematic review}

The University internationalization processes are an adaptive response to a global context marked by globalization, which involves roughly greater political, economic and social interaction among countries and regions. In this context, it is valid to ask what is the role and contributions of the academic library in the internationalization of the campus, considering that historically, its main purpose has been to support the university in the development of its functions. To find answers, a systematic review and a bibliometric analysis of the literature published in Information Science between 2005 and 2015 were performed. A continued growth in published research, led by librarians from the United States, China and Canada, was found. They address different aspects of library work that support both transnational education and internationalization 'at home': supporting study-abroad students and programs, information literacy, collection development, studies on the influence of cultural and linguistic differences in communication and services, among others. Recommendations for library practice related to management strategies, services, infrastructure, training and research, were identified. 
Keywords: Internationalization; Higher Education; Academic Library; Librarian practices.

\section{Contribuições da biblioteca universitária à internacionalização do campus: análise sistemática da literatura}

Os processos de internacionalização universitária constituem resposta adaptativa a um contexto mundial marcado pela globalização, que implica, grosso modo, maior inter-relação política, econômica e social entre países e regiões. Neste contexto, é válido perguntar-se quais são o papel e as contribuições da biblioteca universitária nos processos de internacionalização do campus, tendo em conta que, historicamente, sua razão de ser tem sido servir de apoio à universidade no desenvolvimento de suas funções. Para encontrar respostas, se realizou uma revisão sistemática da literatura publicada em Ciência da Informação entre 2005 e 2015 e uma análise bibliométrica dessa produção científica. Constatou-se um crescimento contínuo da investigação publicada, liderada por bibliotecários dos Estados Unidos, China e Canadá, que tratam de diferentes aspectos do trabalho bibliotecário que apoiam tanto a educação transnacional como a internacionalização 'em casa': serviços bibliotecários para apoiar estudantes e programas no exterior, programas de alfabetização informacional, estudos sobre a influência das diferenças culturais e linguísticas na comunicação e serviços, desenvolvimento de coleções, entre outras. Identificaramse recomendações para a prática bibliotecária relacionadas com estratégias administrativas, de serviços, infraestrutura, formação e investigação.

Palavras-chave: Internacionalização; Educação Superior; Bibliotecas Universitárias; Práticas Bibliotecárias.

Recebido em 20.10.2016 Aceito em 11.07.2017 


\section{Introducción}

Enormes transformaciones tecnológicas, económicas, políticas y sociales se han estado produciendo en el mundo en los últimos treinta años, conduciendo a una mayor interconexión entre sectores, organizaciones y países, a una agudización de la competencia por mercados y a una mayor exposición a fuerzas que, por una parte, tienden a homogeneizar culturas, valores e ideologías, y por otra, tienden a reafirmar diferencias e individualidades. Las universidades también se encuentran inmersas en el torbellino de presiones globalizadoras, forzándolas a adaptarse para responder a este entorno dinámico. Las adaptaciones incluyen, entre otras, mayor énfasis en la alineación de la universidad con la industria y las empresas para la producción de conocimientos e innovaciones y en la formación de estudiantes con competencias y habilidades para insertarse en un mercado de trabajo global. En un sentido más general, se requiere formar ciudadanos con competencias para entender y actuar responsablemente en un mundo signado por diferencias culturales y socioeconómicas, con retos relacionados con la justicia, la equidad y la sostenibilidad.

Los procesos de internacionalización constituyen la respuesta adaptativa a este contexto global. Una de las definiciones más conocidas de internacionalización en el sector de la educación superior es la de Knight (2004), que la comprende como el proceso de integrar la dimensión internacional/intercultural a la enseñanza, la investigación, la extensión y la distribución de servicios educativos. Los propósitos y motivaciones detrás de los procesos de internacionalización son variados y dependen también de las características de las instituciones (por ejemplo, si son públicas o privadas). En unos casos las motivaciones están más alineadas con la cooperación internacional, la elevación de la calidad de la enseñanza y la investigación y la formación de "ciudadanos globales"; en otros casos, estos procesos están más dirigidos a lograr posicionamiento en el mercado de servicios educativos y la obtención de lucros.

Dentro de los procesos de internacionalización de la educación superior se reconocen dos modalidades: la educación transnacional y la internacionalización 'en casa' (KNIGHT, 2013). La primera, reúne todos aquellos procesos y actividades que implican movilidad a través de fronteras nacionales (de alumnos, profesores, programas y proveedores). Esta variante, la de mayor tradición y fuerza, tiene como protagonistas principales a los estudiantes internacionales, definidos por la Unesco (2005) como aquellos que han cruzado una frontera nacional para estudiar o están matriculados en un programa de estudios a distancia fuera de su país.

La modalidad de internacionalización 'en casa' implica desarrollar una agenda internacional/intercultural sin salir del país, para los estudiantes y profesores del campus, a través del fomento del intercambio con estudiantes/profesores internacionales; el estudio de lenguas extranjeras; la incorporación de perspectivas 
internacionales/interculturales al currículo y técnicas de enseñanza; el estímulo a diversas formas de colaboración en la investigación; el uso de herramientas para educación a distancia; la atención a las dinámicas internas en aulas "internacionales", así como el fomento de la sensibilidad y responsabilidad de estudiantes y profesores ante temas internacionales (HARRISON; PEACOCK, 2010).

En este contexto, parece válido preguntarse cuál es el papel de la biblioteca universitaria en los procesos de internacionalización del campus, teniendo en cuenta que, históricamente, su razón de ser ha sido servir de apoyo a la universidad en el desarrollo de sus funciones (docencia, investigación, difusión cultural), alineada a su misión y objetivos.

El estudio de Sánchez-Tarragó, Bufrem, Santos (2016), que exploró las dimensiones más relevantes que relacionan la Ciencia de la Información y la Bibliotecología con la internacionalización de la educación superior, constató el crecimiento de la literatura que aborda el papel de las bibliotecas universitarias en diferentes aristas de la internacionalización, tanto aquellas que contribuyen a la internacionalización 'en casa' como con las variantes de educación transnacional. No obstante, aunque en teoría este papel de la biblioteca universitaria en los procesos de internacionalización se considera coherente con la misión histórica de esta institución, estudios recientes muestran que muchas veces ni la literatura sobre internacionalización de la educación superior incluye a las bibliotecas, ni estas estas contempladas en los planes y estrategias de internacionalización de las universidades (WITT; KUTNER; COOPER, 2015).

La presente investigación se propone profundizar en el análisis de las contribuciones de la biblioteca universitaria a la internacionalización del campus, a través de la caracterización de la comunidad académica que investiga sobre el tema, las temáticas más abordadas en esta línea de investigación, así como la identificación y sistematización de las contribuciones y las recomendaciones para la práctica profesional.

\section{Métodos}

Para esta investigación se combinó el método de revisión sistemática de la literatura, con técnicas bibliométricas. Los métodos seleccionados permitieron, por una parte, realizar una revisión exhaustiva de la literatura existente para la identificación de las contribuciones de la biblioteca universitaria con los procesos de internacionalización, así como la extracción de recomendaciones para la práctica, y por otra, caracterizar cuantitativa y cualitativamente la comunidad académica que investiga el tema y su producción científica.

Xu, Kang y Song (2015) definen una revisión sistemática como un método de investigación riguroso, transparente y reproducible, que incluye el establecimiento de preguntas de investigación específicas, la formulación de criterios de inclusión/exclusión y la evaluación cualitativa de los estudios incluidos. Constituyen un método útil para reunir todos los 
datos pertinentes sobre un tema específico y sirven para cuestionar y sintetizar los resultados de un determinado informe. Su propósito, generalmente, es determinar lo que se sabe y no se sabe sobre determinado tema y cuáles son las recomendaciones para la práctica (GRANT; BOOTH, 2009).

La revisión sistemática implica el establecimiento de varias etapas (PHELPS; CAMPBELL, 2012):

1-definición de una o varias preguntas de investigación específicas;

2-definición de criterios de inclusión y exclusión;

3-búsqueda exhaustiva de estudios pertinentes;

4-análisis preliminar de los resultados a través de la aplicación de criterios de inclusión y exclusión;

5-extracción de datos guiados por preguntas de investigación y objetivos;

6-análisis, síntesis y presentación de los datos dependiendo de los datos extraídos de cada estudio.

Las técnicas de análisis bibliométricas, por su parte, definidas como la aplicación de métodos matemáticos y estadísticos al estudio de la literatura científica (PRITCHARD, 1969), han sido ampliamente usadas en los últimos sesenta años para medir el uso de los documentos científicos y los resultados de la investigación en los diferentes campos del conocimiento, así como caracterizar las comunidades científicas, identificar los líderes en dominios del conocimiento y detectar tendencias en la investigación.

El proceso de investigación se realizó siguiendo las etapas definidas por Phelps y Campbell (2012) para la revisión sistemática e introduciendo una etapa intermedia de análisis bibliométrico que permitió caracterizar la comunidad científica y su producción, para posteriormente realizar el análisis sistemático de la investigación publicada (Figura 1). 
Figura 1 - Proceso de selección de documentos para el análisis
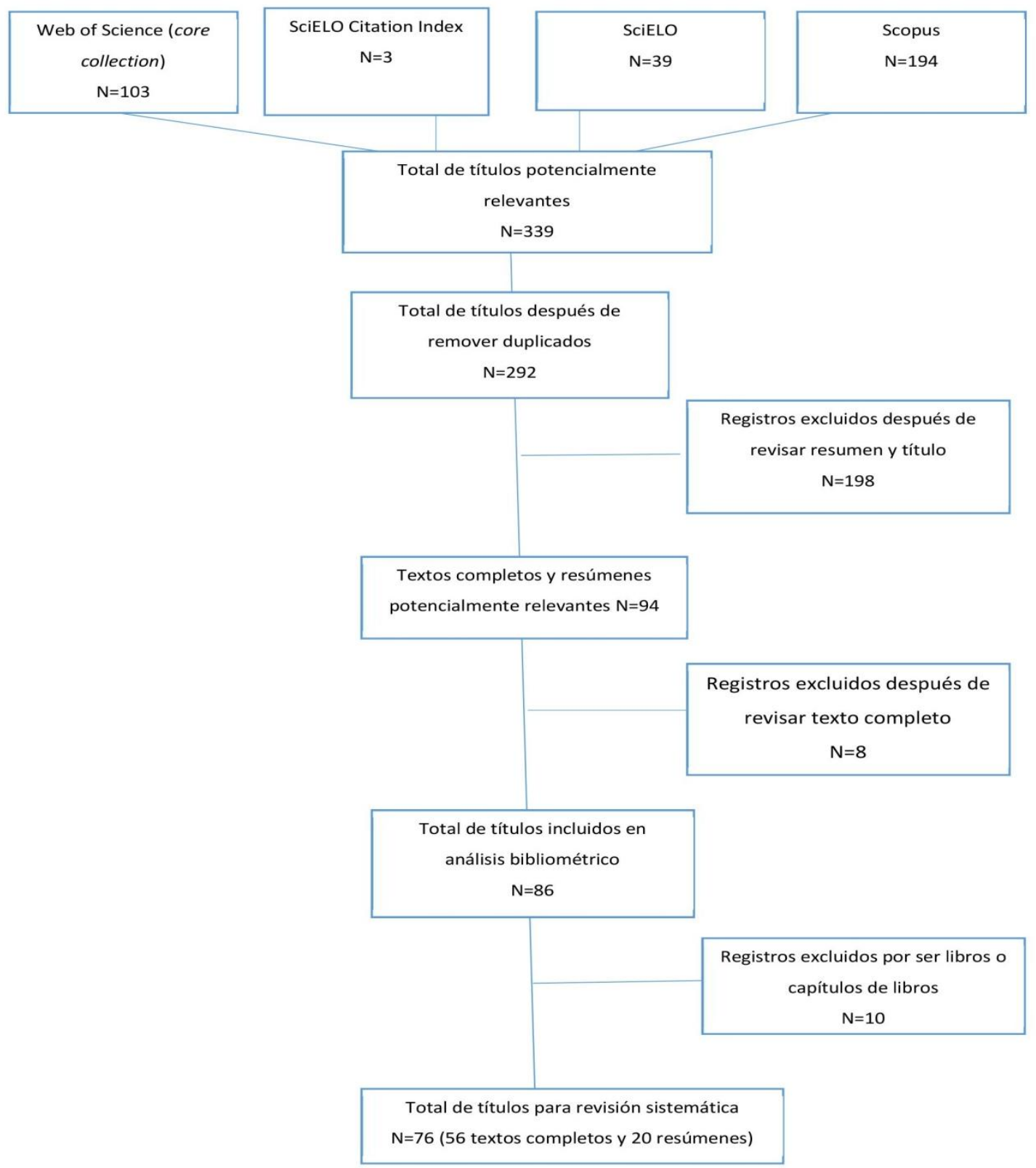

Fuente: Las autoras (2016).

Las preguntas de investigación que guiaron el análisis fueron: ¿cuáles son las características de la comunidad científica que publica los trabajos más relevantes sobre la contribución de la biblioteca académica a la internacionalización de la Educación Superior? ¿Cuáles son los temas más investigados? ¿Cuáles son las actividades o funciones con las que contribuyen las bibliotecas a la internacionalización? ¿Qué lecciones o recomendaciones pueden extraerse para la práctica profesional?

Se utilizaron como fuente de datos las bases de datos Scopus, Web of Science (WoS), SciELO Citation Index y SciELO. Se emplearon las siguientes estrategias de búsqueda en el período de 2005 a 2015: 
(internationalization OR internationalisation) AND librar*; (internationalization OR internationalisation) AND "information science"; "international students" AND librar*; "study abroad" AND librar*; "international cooperation" AND "academic library"; "cross-border education" AND librar*; "transnational education" AND librar*. Las búsquedas se realizaron en abril de 2016. Los resultados de las búsquedas fueron procesados en Vantage Point 9.0. y tras el análisis de títulos, resúmenes y textos completos se descartaron duplicados y documentos temáticamente no relevantes. Las búsquedas en las bases de datos SciELO Citation Index y SciELO no arrojaron resultados relevantes. En el Cuadro 1 se muestran las variables utilizadas para los análisis.

Cuadro 1 - Variables utilizadas en el estudio

\begin{tabular}{|c|c|}
\hline \multicolumn{2}{|r|}{ Análisis bibliométrico } \\
\hline Variables & Definición \\
\hline Cantidad de documentos & $\begin{array}{l}\text { Total de registros sobre Biblioteca Universitaria e } \\
\text { internacionalización }\end{array}$ \\
\hline Tipos de documentos & Cantidad de registros por tipología documental \\
\hline Productividad por fuente & Cantidad de registros publicados por fuente \\
\hline Productividad de Autores & Total de registros por autor \\
\hline Productividad por país & Total de registros por país \\
\hline Productividad por instituciones & Total de registros por instituciones \\
\hline Palabras clave & Total de registros por palabra clave \\
\hline \multicolumn{2}{|l|}{ Revisión sistemática } \\
\hline Variables & Definición \\
\hline Autoría & Simple o colaboración \\
\hline Colaboración & Entre instituciones, entre la misma institución, entre países \\
\hline Técnicas de investigación & Cuestionario, observación, entrevista etc. \\
\hline Población objeto de estudio & Estudiantes, bibliotecarios, profesores etc. \\
\hline País de la investigación & País donde se realiza el estudio \\
\hline País de la población estudiada & País o región de donde procede la población estudiada \\
\hline Teoría o Modelo & Teoría o modelo que sustenta el estudio \\
\hline Temáticas/subtemáticas & Asuntos específicos sobre el que tratan los artículos \\
\hline Implicaciones para bibliotecas & $\begin{array}{l}\text { Contribuciones/Propuestas/recomendaciones/buenas } \\
\text { prácticas/Conclusiones relevantes }\end{array}$ \\
\hline
\end{tabular}

Fuente: Las autoras (2016). 


\section{Resultados y discusión}

\subsection{Caracterización general de la comunidad científica y su producción sobre internacionalización y biblioteca universitaria}

La figura 2 muestra la cantidad de documentos registrados en las bases de datos estudiadas que abordan dimensiones de la relación entre biblioteca universitaria y la internacionalización del campus (86 documentos). La tendencia es al aumento en la producción científica. Aunque los trabajos sobre esta arista habían comenzado desde 1986 como constató el trabajo de Sánchez-Tarragó, Bufrem, Santos (2016), es a partir de 2009 que se observa un aumento importante, con un pico expresivo en 2015.

Figura 2 - Distribución anual de documentos sobre Biblioteca Universitaria e internacionalización (2005-2015)

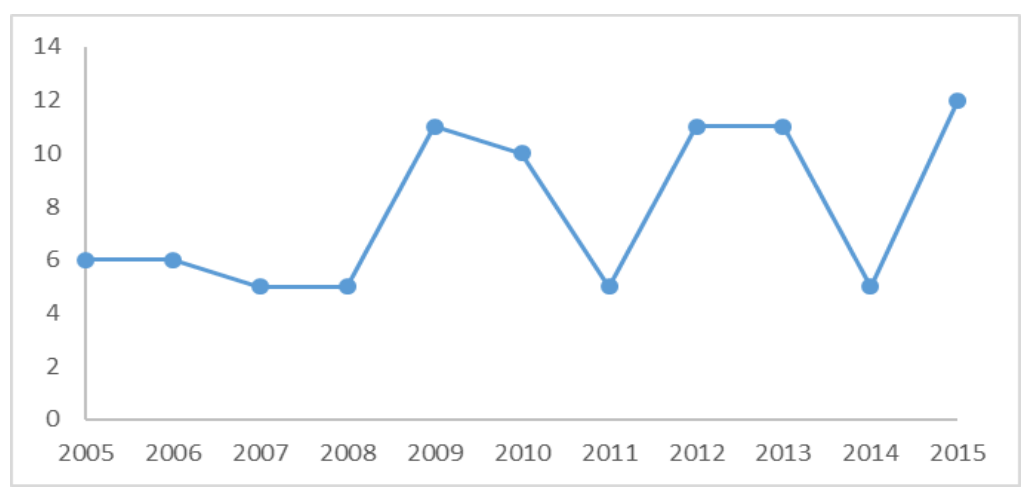

Fuente: Las autoras (2016).

La producción científica ha sido publicada fundamentalmente en artículos de revistas y se encuentra distribuida en 42 fuentes. El $25 \%$ de los artículos fue publicado por dos revistas: Journal of Academic Librarianship y Reference Services Review.

La literatura que compone este corpus fue producida por 167 autores. Los más productivos son Yusuke Ishimura, actualmente en la Edith Cowan University (ECU), Australia; Karen Bordonaro, de la Brock University, Canadá y Laurie Kutner, de la University of Vermont, Estados Unidos, con 4, 3 y 3 trabajos, respectivamente. De los 14 autores con más de 2 trabajos en el corpus, 9 trabajan en universidades de Estados Unidos; la mayoría son autoras; predominan las bibliotecarias y bibliotecarios con funciones relacionadas con la alfabetización informacional o que sirven de enlace a programas de enseñanza de inglés a alumnos extranjeros. Resulta interesante que varios de ellos poseen, además de maestría o doctorado en Ciencia de la Información y Bibliotecología, una formación académica como profesores de inglés para alumnos extranjeros no anglófonos. 
Los autores proceden de 27 países. No obstante, hay una concentración muy expresiva de autores procedentes de Estados Unidos, seguidos a cierta distancia de Canadá, China, Australia y Reino Unido. La región latinoamericana solo está representada por 2 trabajos de México. Se identificaron 107 instituciones. De ellas, las más productivas son Pennsylvania State University y University of Illinois, ambas de Estados Unidos, que aportaron 4 trabajos cada una. Las universidades Brock y McGill, de Canadá y Vermont, de Estados Unidos, produjeron 3 trabajos cada una. El $81 \%$ de las instituciones fue responsable por un solo documento. Estos resultados son consistentes con los obtenidos por Sánchez-Tarragó, Santos, Bufrem $(2014,2015)$ respecto a los mayores productores de literatura sobre internacionalización de la educación superior: Estados Unidos, Reino Unido y China. Estos países son también los líderes en el mercado de servicios educativos transnacionales; datos del Instituto de Educación Internacional revelan que Estados Unidos, Reino Unido y China reciben el $24 \%$, $11 \%$ y $8 \%$, respectivamente, del flujo de estudiantes internacionales del mundo (INSTITUTE FOR INTERNATIONAL EDUCATION - IIE, 2015a).

En el caso de la región Latinoamericana, el informe del 2014 de la Asociación Internacional de Universidades indicaba que en los últimos años se ha producido un mayor interés gubernamental en las políticas y estrategias de internacionalización, con un aumento del financiamiento público para apoyar estos esfuerzos que se manifiesta, sobre todo, en un aumento de la movilidad de estudiantes. No obstante, con respecto a otras regiones, se encuentra rezagada en relación a políticas de internacionalización, financiamiento, movilidad estudiantil y del cuerpo docente, internacionalización del currículo y estrategias organizativas (INTERNATIONAL ASSOCIATION OF UNIVERSITIES - IAU, 2014). Aun cuando se reconoce un aumento de la producción científica regional sobre este tema "los especialistas latinoamericanos reconocidos y citados por sus pares son pocos y los grupos expertos son escasos y muchas veces estructurados en torno a algunos líderes" (DIDEU-AUPETIT, 2014, p. 14). Una situación similar pudiera estarse manifestando en la producción sobre el tema en áreas especializadas como la Bibliotecología y Ciencia de la Información.

A través del análisis sistemático de los 74 artículos de revista y 2 trabajos de eventos científicos pudieron detectarse otras características de la comunidad investigadora y las investigaciones realizadas. El análisis de los artículos muestra que más de la mitad de los trabajos (58\%) fue producida en colaboración, lo que sugiere, tal como señala el estudio de Click, Wiley e Houlihan (2016), que las Ciencias de la Información han adoptado prácticas más colaborativas en los últimos 25 años. No obstante, el $60 \%$ de estos trabajos fue elaborado en coautoría entre miembros de la misma institución y sólo fueron producidos entre autores de instituciones y países diferentes, un $22 \%$ y $18 \%$, respectivamente.

La mitad de los trabajos del corpus fue realizada en Estados Unidos (38), tomando como objeto de estudio a las bibliotecas universitarias de 
ese país y los estudiantes en movilidad académica. Otros estudios se desarrollaron en Canadá (6), China (5), Australia (4) y en menor número, en otros países. Este hallazgo implica que la mayoría de las experiencias identificadas llevan el sello de la práctica bibliotecaria estadounidense, con sus características específicas, que pudieran no ser automáticamente extrapolables a otros contextos. Las poblaciones más estudiadas fueron los estudiantes internacionales procedentes de Asia (fundamentalmente China y Japón). Según el Instituto de Educación Internacional, China está en el número 1 del ranking de emisores de estudiantes internacionales hacia Estados Unidos (58\% en 2014-2015), seguidos por India, Corea del sur y Arabia Saudita (IIE, 2015b).

El $34 \%$ de los estudios son levantamientos de datos que utilizan fundamentalmente cuestionarios en línea e indagan sobre percepciones, actitudes, preferencias y comportamientos de estudiantes y bibliotecarios. Aun cuando este tipo de investigación tiene valor para identificar aspectos relevantes para prácticas y contextos específicos, pudiera no ser de aplicabilidad general. Se requerirían estudios más generales y multicéntricos, incluyendo la elaboración de guías de buenas prácticas. Otras técnicas de investigación utilizadas en menor medida son las entrevistas, grupos focales, observación, diario online, research portfolios y flowcharts.

\section{2 ¿Qué se investiga sobre biblioteca universitaria e internacionalización?}

Las temáticas y subtemáticas abordadas en la investigación sobre biblioteca universitaria e internacionalización fueron exploradas primeramente a través de una matriz de co-ocurrencia de palabras clave (Figura 3). Esta técnica identifica la frecuencia en que pares de palabras aparecen juntas en un cuerpo textual, indicando, a mayor frecuencia de ocurrencia conjunta, mayor afinidad conceptual (MIGUEL; CAPRILE; JORQUERA-VIDAL, 2008).

Figura 3 - Biblioteca Universitaria e internacionalización. Matriz de coocurrencia de palabras clave (2005-2015)

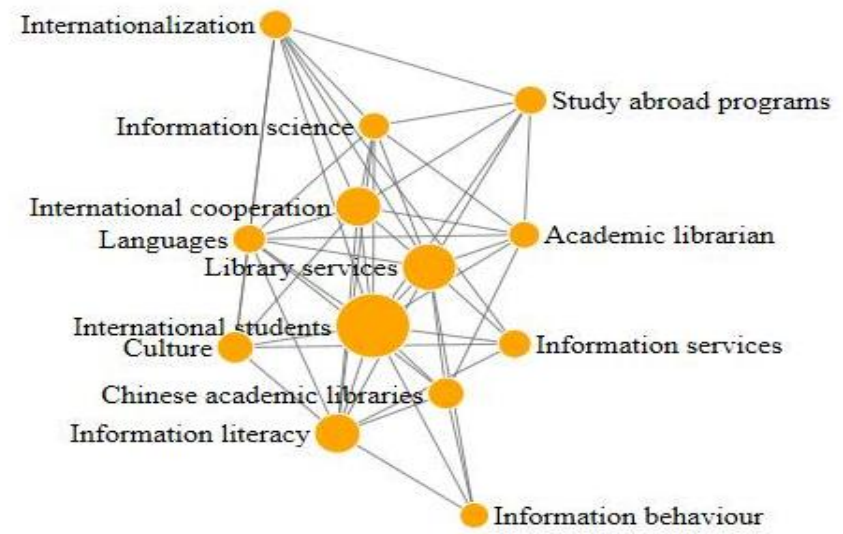

Fuente: Las autoras (2016). 
En esta visualización se puede apreciar tanto el peso de las temáticas representadas por las palabras clave (tamaño del círculo) como las relaciones de afinidad (cercanía) entre ellas. Además de International students, que fue uno de los términos utilizados en la estrategia de búsqueda, se destaca, por ejemplo, Information literacy y Library services lo que sugiere la importancia de estas dimensiones en la relación de la biblioteca universitaria con los procesos de internacionalización. Otros términos, que dan idea de los aspectos críticos en la temática, son representados por la frecuencia de las palabras clave Culture y Language, elementos que marcan la interacción satisfactoria o no entre los sistemas bibliotecarios y los usuarios internacionales.

Resulta interesante el destaque del término Chinese academic libraries, lo que se explica por la enorme importancia que los autores de ese país están otorgando a los procesos de internacionalización. China no es sólo un emisor de estudiantes internacionales sino que también ha experimentado un gran crecimiento como receptor de estudiantes procedentes, fundamentalmente, de Corea del Sur, Estados Unidos, Tailandia, Rusia y Japón (IIE, 2015a). La visualización también permite apreciar cierta distancia entre el término Study abroad program con otros centralizados alrededor de International students, lo cual sugiere una diferenciación en la literatura y en las prácticas entre las dos modalidades de internacionalización: la educación transnacional y la internacionalización 'en casa', aun cuando deben considerarse como dos caras de la misma moneda.

El análisis sistemático posterior, a través de la lectura de los textos completos, permitió la clasificación temática de los trabajos en cinco grandes grupos (Tabla 1), así como otras subclasificaciones temáticas dentro de cada grupo. Este análisis refinado permitió identificar algunos ejemplos relevantes de cómo las bibliotecas universitarias están contribuyendo a los procesos de internacionalización en sus modalidades de internacionalización 'en casa' y educación transnacional, así como recomendaciones para la práctica, que serán presentados en los epígrafes siguientes.

Tabla 1 - Clasificación de los documentos según temáticas

\begin{tabular}{l|c}
\hline Grupo temático & Cantidad de documentos \\
\hline \hline Estudios de usuarios & 29 \\
\hline Servicios de información & 15 \\
\hline Alfabetización informacional & 13 \\
\hline Comunicación y Lenguaje & 11 \\
\hline Competencias y entrenamiento de bibliotecarios & 8 \\
\hline Total & $\mathbf{7 6}$ \\
\hline
\end{tabular}

Fuente: Las autoras (2016). 


\subsection{Contribuciones de la biblioteca universitaria a los procesos de internacionalización}

Las actividades y funciones de las bibliotecas universitarias que contribuyen a los procesos de internacionalización del campus se corresponden en esencia con las funciones básicas de una biblioteca universitaria de apoyar la formación, la docencia y la investigación. Sin embargo, a nuestro modo de ver, ellas implican en primer lugar identificar las especificidades que conllevan los procesos de internacionalización, caracterizados por diferentes niveles de interacción entre individuos con culturas y patrones cognitivos diversos; con necesidades de información influenciadas por los desplazamientos físicos; con competencias y habilidades marcadas por la cultura y los sistemas educativos de procedencia, entre otras consideraciones.

En muchos de estos escenarios ocurre el llamado choque cultural, un conjunto de reacciones tanto afectivas como cognitivas que se producen cuando el individuo entra en contacto por primera vez con culturas y lenguas diferentes a la suya, que pueden desencadenar emociones de miedo, inseguridad, desconfianza y ansiedad. El choque cultural puede constituir un obstáculo importante para el aprovechamiento académico de los estudiantes y su integración social al nuevo ambiente. Estos estudiantes pudieran experimentar también lo que se conoce como ansiedad bibliotecaria (library anxiety), referida como una reacción negativa, una "barrera psicológica", que dificulta el uso y aprovechamiento de los servicios y recursos de la biblioteca. Varios trabajos en este estudio abordan el papel de las bibliotecas y los bibliotecarios ante estos retos, que incluyen el reconocimiento e identificación de estos fenómenos, el entrenamiento de los bibliotecarios para lidiar con las diferencias de lenguaje y comunicación y la adopción de estrategias para adecuar servicios y ambientes de manera que la biblioteca pueda, no solo aliviar el choque cultural y la ansiedad bibliotecaria, sino también apoyar con efectividad las actividades de docencia e investigación de este grupo de usuarios (GILTON, 2007; LU; ADKINS, 2012; RAFIK-GALEA; ISHAK; MARJI, 2012; ZHANG, 2006). Aunque la mayoría de los trabajos se basan en las diferencias entre estudiantes de culturas occidentales y orientales ( $y$ a la influencia de las diferencias en patrones de pensamiento y comunicación no verbal según sean culturas individualistas/colectivistas, y de contexto bajo/contexto alto), las implicaciones para la biblioteca pueden generalizarse a cualquier estudiante extranjero. De hecho, estos fenómenos tienen influencia en los estudios de usuario, de percepciones y satisfacción con la biblioteca, los servicios de información y los programas de alfabetización informacional

Varios trabajos revelan que los estudiantes internacionales tienen necesidades de información muy variadas, que van más allá de sus necesidades de información académica. Este grupo necesita realizar numerosos ajustes en su vida diaria y enfrentar retos para adaptarse al país extranjero por lo que sus necesidades de información pueden ir desde 
transporte, entretenimiento, alquileres, sistema legal, entre otros muchos otros tópicos (SACKERS; SECOMB; HULETT, 2008; SIN, 2015). Por tanto, los estudios sobre las preferencias y necesidades de información de los estudiantes internacionales, sus comportamientos de información, los patrones de uso de las bibliotecas y las percepciones de estos usuarios sobre las bibliotecas y los bibliotecarios, constituyen puntos de partida importantes para propiciar la integración exitosa de estos estudiantes no solo a un nuevo contexto académico sino también al ambiente social y cultural, a veces muy diferente de aquel de donde provienen.

Por otra parte, las búsquedas de información que realizan los estudiantes internacionales y el uso de la biblioteca está influenciado por varios factores como la proficiencia en la lengua que se habla en el país destino, las habilidades de los estudiantes en el uso de las tecnologías y su experiencia anterior con la biblioteca en su lugar de origen, como constatan varios trabajos, entre ellos, los de Hughes (2010), Jiao et al. (2009) y Shao et al. (2013). Otros estudios muestran que muchos estudiantes internacionales, sobre todo aquellos cuya lengua materna difiere de la lengua del país destino, presentan problemas de escritura, lectura y comunicación en la lengua extranjera, lo cuales, sumado a la falta de habilidades para el manejo de la información científica, constituyen trabas para su aprovechamiento docente. En esta situación se basan varios trabajos que proponen programas de alfabetización informacional específicos para usuarios internacionales (HURLEY; HEGARTY; BOLGER, 2006; LAHLAFI; RUSHTON, 2015). Resulta interesante también el énfasis en los programas dedicados a combatir el plagio y otras malas prácticas durante la docencia y la investigación, las cuales muchas veces están influenciadas por complejos factores culturales, educacionales y lingüísticos, así como formar competencias en investigación, citación y redacción científica (AMSBERRY, 2009; GUNNARSSON; KULESZA; PETTERSSON, 2014; ZIMERMAN, 2012).

Diversos estudios abordan la necesidad de adecuar las colecciones y servicios de información para satisfacer las necesidades de estos grupos de usuarios, lo que incluye, por ejemplo, la creación de sitios web de bibliotecas bilingües 0 multilingües, de colecciones de recursos multiculturales, la adecuación de los ambientes a través de señalizaciones apropiadas, la organización de espacios de interacción ajustados a las características de estos usuarios e incluso la identificación de los medios de comunicación más utilizados por los estudiantes internacionales para emplearlos en la promoción de los servicios y la comunicación con la biblioteca (AIHONG, 2009; SAW et al., 2013; SCHOMBERG; GRACE, 2005; TORRAS; VAAGAN, 2006).

Un aspecto bastante tratado en este corpus es la adecuación de los servicios de referencia a esta dimensión internacional. Así, algunos trabajos discuten la influencia de las barreras lingüísticas y culturales en la efectividad del servicio ofrecido (ADEMODI, 2011; YE, 2009); otros, son propuestas de servicios de referencia ajustados para los usuarios internacionales. Es interesante el desarrollo de toda una línea de trabajos 
enfocados, no solo a los usuarios internacionales que vienen a la biblioteca, sino a los estudiantes y profesores que viajan a estancias de estudio al exterior. Estos, en muchos casos, están imposibilitados de usar las bibliotecas de la universidad que los acoge y, por tanto, pudieran tener necesidades de información insatisfechas. Algunos estudios en este corpus muestran iniciativas en este sentido que se llevan a cabo utilizando sistemas de teleconferencia y otras tecnologías de comunicación (CHAN et al., 2015; COHEN; BURKHARDT, 2010; KUTNER, 2010).

Otros artículos se refieren al papel de la biblioteca para contribuir al desarrollo de competencias en lenguas extranjeras, por ejemplo, a través de actividades típicas de las bibliotecas como búsquedas en bases de datos (BORDONARO, 2006; 2010) y a la colaboración entre bibliotecarios y profesores en el diseño de cursos para educación transnacional (GRINER; HERRON; WHITE, 2015).

\subsection{Recomendaciones prácticas recogidas en la literatura}

Las contribuciones reseñadas brevemente en el epígrafe anterior requieren de transformaciones tanto a nivel de misión y objetivos de la biblioteca universitaria, como de su infraestructura, colecciones, servicios y formación de sus recursos humanos. Varios trabajos incluidos en este estudio muestran que generalmente las iniciativas de internacionalización son lideradas por las autoridades universitarias y que, en muchos casos, los niveles de internacionalización que las universidades declaran no se corresponden con los niveles que exhiben las bibliotecas. Además, aunque las bibliotecas realizan numerosas actividades que contribuyen con la internacionalización, estas no están articuladas en sus planes estratégicos (BORDONARO; RAUCHMANN, 2015; WITT; KUTNER; COOPER, 2015).

No obstante, los estudios dedicados a explorar las percepciones, actitudes y experiencias de los bibliotecarios sobre los servicios para usuarios internacionales mostraron que estos profesionales están interesados en mejorar los servicios para este grupo de usuario y que en muchos casos reconocen los retos relacionados con la comunicación, las diferencias entre sistemas bibliotecarios y en las prácticas académicas y de investigación de los usuarios provenientes de contextos diversos. Los bibliotecarios, por tanto, reconocen la necesidad de más entrenamiento en lenguas extranjeras, de mejorar las competencias interculturales y la necesidad de diseñar o adaptar colecciones y servicios para este grupo de usuarios. El intercambio internacional bilateral de bibliotecarios también puede contribuir a ampliar la creatividad, promover la innovación y desarrollar competencias interculturales útiles para desarrollar servicios de apoyo a la internacionalización del campus (ISHIMURA; BARTLETT, 2014; SMITH, 2015; SOMERVILLE et al., 2015).

El análisis sistemático de la literatura permitió extraer y sintetizar 66 recomendaciones que pudieran contribuir tanto a articular los esfuerzos de las bibliotecas universitarias en la mediación entre la universidad y los procesos de internacionalización, como a hacer más efectivos sus servicios 
para estudiantes y profesores involucrados en estos procesos. Algunas de ellas se exponen a continuación clasificadas en cinco grupos: estrategias administrativas, formación de bibliotecarios, servicios de información, infraestructura y colecciones, e investigación. Posteriormente se realiza un comentario sobre la necesidad de contextualización de estas recomendaciones.

\subsubsection{Estrategias administrativas}

a)Incorporar y articular las funciones de la biblioteca universitaria al plan estratégico de internacionalización de la universidad.

b)Realizar cambios en la misión y en el plan estratégico de la biblioteca de manera que incluya los procesos y actividades que apoyen y promuevan la internacionalización del campus.

c)Fomentar la participación de bibliotecarios en los comités de internacionalización del campus $y$ en organizaciones internacionales.

d)Mantener y desarrollar canales de comunicación con la unidad administrativa responsable de los estudiantes internacionales y otras redes internacionales de estudiantes. e)La colaboración podría incluir proporcionar información cruzada entre los sitios web de las instituciones y participación de la biblioteca en reuniones de orientación con los estudiantes internacionales.

f)Establecer alianzas estratégicas con las instituciones académicas en el exterior que acogen a los estudiantes.

\subsubsection{Formación de bibliotecarios}

a)Establecer la capacitación continua de los bibliotecarios con el objetivo de mejorar su desempeño profesional, el dominio de otros idiomas y las competencias interculturales.

b)Desarrollar en conjunto con otros departamentos de la universidad (Psicología, Educación, Núcleos de Lengua extranjera) entrenamientos para que los bibliotecarios aprendan a reconocer el impacto de los patrones de pensamiento y las diferencias culturales en los estilos de comunicación y aprendizaje.

c)Integrar la comunicación intercultural en el currículo de Ciencia de la Información.

d)Estimular en los bibliotecarios la ampliación de sus conocimientos y lecturas sobre la historia, cultura y sistemas de educación de otros países, además de estudios comparados en Bibliotecología y Ciencia de la Información, que permitirán 
conocer las características de los usuarios extranjeros y las tradiciones y prácticas de sus bibliotecas.

e)Promover la movilidad de bibliotecarios y el intercambio con colegas de otras instituciones nacionales e internacionales.

\subsubsection{Infraestructura y colecciones}

a)Facilitar un ambiente adecuado en la biblioteca que favorezca la comunicación y empatía entre los usuarios internacionales y bibliotecarios.

b)Proporcionar en la biblioteca un sistema de señales bilingüe, o multilingüe, que auxilie a los usuarios en la identificación y localización de locales y servicios.

c)Preparar información multilingüe sobre la naturaleza y propósitos de la biblioteca, las funciones de los bibliotecarios y los términos típicos de la biblioteca.

d)Incluir en las colecciones de las bibliotecas materiales multiculturales y multilingües de acuerdo con la procedencia típica de los usuarios internacionales.

e)Apoyar a los estudiantes y profesores en estancias en el extranjero con bibliografía actualizada sobre los diferentes temas de investigación, a través de la creación de colecciones digitales disponibles en línea.

Realizar articulaciones entre la biblioteca y redes de estudiantes residentes en el país y en el extranjero, que fomente el intercambio de información.

\subsubsection{Servicios de información}

a)Realizar estudios para identificar necesidades, percepciones y experiencias de uso de servicios de información de estudiantes y profesores internacionales, incluyendo necesidades de alfabetización informacional.

b)Diseñar servicios de información acordes a las necesidades de los usuarios internacionales, que incluya información académica y de uso cotidiano.

c)Diseñar las interfaces de los OPACs y sitios web, teniendo en cuenta las especificidades de los usuarios internacionales, sobre todo relacionados con las barreras de lenguaje.

d)Impartir sesiones de entrenamiento básicas sobre la biblioteca y sus servicios para los estudiantes que entran al primer semestre. 
e)Identificar los medios de comunicación preferidos por los estudiantes internacionales y explotar el potencial de estos para a comunicación y diseminación de información.

f)Promover oportunidades para la interacción informal entre el equipo de bibliotecarios y los usuarios internacionales.

g)Definir indicadores de evaluación de la calidad de los servicios que se brindan en la biblioteca y el desempeño de los bibliotecarios, donde se incluyan indicadores relacionados con usuarios internacionales $y$ otros procesos de internacionalización.

h)Colaborar con profesores e investigadores en la internacionalización de los currículos y el diseño de investigaciones colaborativas.

\subsubsection{Investigación}

a)Realizar estudios que identifiquen las percepciones de los bibliotecarios sobre los servicios para usuarios internacionales, así como sus necesidades de entrenamiento.

b)Realizar estudios que caractericen la población de estudiantes y otros usuarios internacionales y sus necesidades de información.

c)Promover la realización de proyectos colaborativos donde participen bibliotecarios, profesores y estudiantes internacionales.

d)Fomentar la investigación en los bibliotecarios que les permita ampliar sus conocimientos sobre historia, cultura, sistemas de educación de otros países y prácticas de sus bibliotecas.

e)Realizar estudios que permitan comparar las experiencias y enfoques de internacionalización de las bibliotecas universitarias de diferentes países y la elaboración de guías de buenas prácticas.

A pesar del valor de estas recomendaciones, vale la pena retomar las palabras de Arenas Franco, Covarrubias Escobar e Anania Garib (2002, p. 163) cuando plantean que "cada sistema de bibliotecas es único y parte de su universidad, por lo tanto, debe funcionar dentro de su cultura y responder a sus necesidades". Esto es particularmente importante si tenemos en cuenta que más de la mitad de los trabajos se desarrollaron en bibliotecas universitarias de Estados Unidos, inmersas en un contexto en el que prevalece generalmente un modelo de internacionalización 
dirigido a la obtención de lucros y al posicionamiento en el mercado de servicios educativos, y cuyas características y funciones pudieran no corresponderse con otras bibliotecas en otras partes del mundo.

Al respecto, Bordonaro y Rouchman (2015) destacan varias diferencias entre Norteamérica (Estados Unidos y Canadá) y Alemania (que pudieran extenderse a otros países de Europa continental) con respecto a sus percepciones y actitudes sobre la internacionalización y el papel de las bibliotecas universitarias. Además de las diferencias respecto a las motivaciones lucrativas de la internacionalización existentes en los sistemas universitarios norteamericanos y ausente en Alemania y otros países de Europa continental, las autoras señalan, por ejemplo, el papel activo de los bibliotecarios de Norteamérica en el desarrollo del currículo, lo cual no se considera responsabilidad de las bibliotecas en el caso alemán; la idea de que los estudiantes internacionales son académicamente deficientes, debido a que no son anglófonos nativos ( $y$ que por ello requieren apoyo adicional) tampoco es prevalente en las bibliotecas universitarias alemanas; y la existencia más antigua de oficinas de servicios a estudiantes internacionales en universidades de Norteamérica, respecto a la novedad que representa para el contexto universitario alemán. Según las autoras, estas diferencias contribuyen a que la internacionalización en las bibliotecas alemanas muchas veces esté implícita dentro de sus procesos y servicios bibliotecarios y no constituya, como en el caso de las bibliotecas de Norteamérica un objetivo explícito que requiera ser ampliamente divulgado y exteriorizado a través de todos los medios posibles.

Algunas diferencias entre las estrategias de internacionalización asumidas por bibliotecas occidentales y orientales (en este caso de China) también emergieron en el estudio de Shao y Scherlen (2011). En su análisis sobre percepciones y actitudes de bibliotecarios chinos con relación a los servicios dedicados a estudiantes y profesores internacionales quedó claro el interés de los bibliotecarios chinos en perfeccionar los servicios para este grupo de usuarios, pero también su cautela acerca de la necesidad de crear estructuras formales, tales como comités, para alcanzar esos objetivos. Estos análisis comparativos invitan a realizar reflexiones a la hora de adoptar estrategias foráneas.

\section{Consideraciones finales}

La internacionalización de la educación superior, conceptualmente más abarcadora que la noción de cooperación internacional, constituye un proceso continuo de integración de la dimensión internacional e intercultural en los procesos de aprendizaje, investigación y servicios educativos, que se considera actualmente parte de la definición de calidad en educación e investigación. No constituye un fin en sí misma, sino un medio para, en unos casos, lograr la excelencia académica, fomentar el entendimiento intercultural o global y preparar a los estudiantes para un 
mundo interconectado; en otros casos, un medio para posicionarse en el mercado de bienes y servicios educativos.

La biblioteca universitaria, cuya razón de ser es servir de apoyo a la universidad en el desarrollo de sus funciones, articulándose a su misión, fines y objetivos, debería desempeñar un papel protagónico en el proceso de internacionalización universitario. A tono con el incremento de la investigación y la implementación de políticas universitarias en este sentido, también se manifiesta un creciente interés en estos tópicos por parte de las bibliotecas. El método utilizado para esta investigación, que combina la revisión sistemática de la literatura con técnicas bibliométricas, permitió identificar y reflexionar sobre las principales contribuciones de las bibliotecas a los procesos de internacionalización. Para esto fueron establecidas preguntas de investigación específicas, se formularon criterios para la búsqueda, selección, recogida y clasificación de los documentos y se pudieron establecer las relaciones más importantes entre los diferentes tópicos.

La caracterización de la producción científica sobre internacionalización de la educación superior y bibliotecas universitarias realizada en este trabajo mostró un crecimiento sostenido de las investigaciones sobre el tema, que se ha reforzado en los últimos diez años, con un protagonismo de bibliotecarios e investigadores de Estados Unidos, Canadá, China, Reino Unido y Australia. En América Latina y el Caribe son escasas aún las investigaciones publicadas sobre el tema, lo cual puede ser reflejo también de incipiente participación regional en estos procesos, a pesar de los avances desiguales en los últimos años.

La contribución de la biblioteca universitaria a la internacionalización del campus implica apoyar todos aquellos procesos que favorecen la integración de los estudiantes y profesores en movilidad a la dinámica universitaria, tanto desde el punto de vista académico como social; estimular y viabilizar el intercambio y difusión de información tanto entre los miembros del campus (ya sean domésticos o foráneos) como nacional e internacionalmente; contribuir a fomentar la sensibilidad intercultural y la adopción de perspectivas globales en la docencia, la investigación y otras actividades universitarias, entre otras muchas. No obstante, las recomendaciones aquí presentadas, sistematizadas a partir del análisis de la literatura publicada, apuntan solo a líneas de acción que deben ser contextualizadas de acuerdo con las características de cada biblioteca y centro universitario, por ejemplo, la cantidad y procedencia de los alumnos y profesores internacionales que recibe o envía a estancias en el exterior, las características de sus programas de estudio, las diferentes modalidades de internacionalización que adoptan, su cultura organizacional y los recursos que posee. En este sentido, resulta muy pertinente la realización de estudios locales sobre las vías de participación de la biblioteca universitaria en la internacionalización de la educación superior, de modo que las estrategias internacionales puedan contextualizarse y adaptarse. 


\section{Referencias}

ADEMODI, O. Reference service in academic libraries: accommodation of international students. Library Philosophy and Practice, n.1, 2011. Disponível

em: $<$ http://digitalcommons.unl.edu/cgi/viewcontent.cgi?article=1555\&context=libphilprac >. Acesso em: 9 set. 2017.

AIHONG, $F$. Creating a bilingual library information environment for foreign users. The Electronic Library, v. 27, n. 2, p. 237-246, 2009.

AMSBERRY, D. Deconstructing plagiarism: international students and textual borrowing practices. The Reference Librarian, v. 51, n. 1, p. 3144, 2009.

ARENAS FRANCO, M. L; COVARRUBIAS ESCOBAR, V. G; ANANIA GARIB, G. A. Estudio comparativo entre bibliotecas universitarias latinoamericanas y estadounidenses. Revista española de documentación científica, v. 25, n. 2, p. 162-181, 2002.

BORDONARO, K. Language learning in the library: an exploratory study of ESL students. The Journal of Academic Librarianship, v. 32, n. 5, p. 518526, 2006.

BORDONARO, K. Is library database searching a language learning activity? College \& Research Libraries, v. 71, n. 3, p. 273-284, 2010.

BORDONARO, K; RAUCHMANN, S. Internationalization in German Academic Libraries: moving beyond North American perspectives. portal: Libraries and the Academy, v.15, n.4, p.677-697, 2015.

CHAN, K. P. et al. Libraries across the sea: using a virtual presence and skilled student assistants to serve students abroad. Journal of Library Administration, v. 55, n. 4, p. 278-301, 2015.

CLICK, A. B.; WILEY, W. W.; HOULIHAN, M. The internationalization of the academic library: a systematic review of 25 years of literature on international students. College \& Research Libraries, 2016. Disponível em: <http://crl.acrl.org/content/early/2016/03/22/crl16-877.full.pdf>. Acesso em: 14 jul. 2016.

COHEN, S. F.; BURKHARDT, A. Even an ocean away: developing Skypebased reference for students studying abroad. Reference Services Review, v. 38, n. 2, p. 264-273, 2010.

DIDOU-AUPETIT, S. La Internacionalización de la educación superior y la ciencia en América Latina: un balance necesario. In: DIDOU-AUPETIT, D; JARAMILLO DE ESCOBAR, V. (Org.). Internacionalización de la educación superior y la ciencia en América Latina: un estado del arte. Caracas: Unesco-IESALC, 2014, p. 9-15.

GILTON, D.L. Culture shock in the library: Implications for information literacy instruction. Research Strategies, v. 20, n. 5, p. 424-432, 2007. 
GRANT, M. J; BOOTH, A. A typology of reviews: an analysis of 14 review types and associated methodologies. Health Information and Libraries Journal, v. 26 n. 2, p. 91-108, 2009.

GRINER, L.; HERRON, P. J.; WHITE, S. Study abroad partnerships: librarians, business faculty, and in-country facilitator develop an innovative experiential learning program. Journal of Business \& Finance Librarianship, v. 20, n. 3, p. 189-208, 2015.

GUNNARSSON, J.; KULESZA, W. J.; PETTERSSON, A. Teaching international students how to avoid plagiarism: librarians and faculty in collaboration. The Journal of Academic Librarianship, n. 40, p. 413-417, 2014.

HARRISON, N.; PEACOCK, N. Cultural distance, mindfulness and passive xenophobia: using integrated threat theory to explore home higher education students' perspectives on internationalization at home. British Educational Research Journal, v. 36, n. 6, p. 877-902, 2010.

HUGHES, H. International students' experiences of university libraries and librarians. Australian Academic \& Research Libraries, v. 41, n. 2, p. 77-89, 2010.

HURLEY, T.; HEGARTY, N.; BOLGER, J. Crossing a bridge: the challenges of developing and delivering a pilot information literacy course for international students. New Library World, v. 107, n. 7-8, p. 302-320, 2006.

INTERNATIONAL ASSOCIATION OF UNIVERSITIES (IAU). Internationalization of higher education: growing expectations, fundamental values. 4ta global survey. Paris: IAU, 2014.

INSTITUTE FOR INTERNATIONAL EDUCATION (IIE). Project Atlas. 2015 a. Disponível em: <http://www.iie.org/ /media/Files/Services/Website2015/Project-Atlas-Infographic-2015.pdf?la=en>. Acesso em: 14 jul. 2016.

INSTITUTE FOR INTERNATIONAL EDUCATION (IIE). Open Doors Report: fast facts. 2015b. Disponível em: <http://www.iie.org/ /media/Images/Corporate/Other/Open-Doors2015/Infographic-Open-Doors-2015-International-Students.jpg?la=en > . Acesso em: 14 jul. 2016.

ISHIMURA, Y; BARTLETT, J. C. Are librarians equipped to teach international students? A survey of current practices and recommendations for training. The Journal of Academic Librarianship, v. 40, n. 3, p. 313-321, 2014.

JIAO, S. et al. Chinese academic libraries from the perspective of international students studying in China. The International Information \& Library Review, v. 41, n. 1, p. 1-11, 2009. 
KNIGHT, J. Internationalization remodeled definitions, rationales, and approaches. Journal for Studies in International Education, v. 8, n. 1, p.5$31,2004$.

KNIGHT, J. The changing landscape of higher education internationalization: for better or worse? Perspectives: Policy and Practice in Higher Education, v. 17, n. 3, p. 84-90, 2013. Disponível em: <http://dx.doi.org/10.1080/13603108.2012.753957>. Acesso em: 14 jul. 2016.

KUTNER, L. Study Abroad programs: a golden opportunity for academic library engagement. Journal of Library Administration, v. 50, p. 767-778, 2010.

LAHLAFI, A; RUSHTON, D. Engaging international students in academic and information literacy. New Library World, v. 116, n. 5-6, p. 277-288, 2015.

LU, Y; ADKINS, D. Library anxiety among international graduate students. Proceedings of the American Society for Information Science and Technology, v. 49, n. 1, p. 1-4, 2012.

MIGUEL, S.; CAPRILE, L.; JORQUERA-VIDAL, I. Análisis de co-términos y de redes sociales para la generación de mapas temáticos. El Profesional de la Información, v. 17, n. 6, p. 637-646, 2008.

PHELPS, S.F; CAMPBELL, N. Systematic reviews in theory and practice for library and information studies. Library and Information Research, v. 36, n. 112 , p. 6-15, 2012.

PRITCHARD, A. Statistical bibliography or bibliometrics? Journal of Documentation, v. 25 n. 4, p. 348-349, 1969.

RAFIK-GALEA, S.; ISHAK, W. I; MARJI, A. B. Library counter talk: communication encounters between counter staff and international students. Procedia-Social and Behavioral Sciences, v. 66, p. 17-28, 2012.

SACKERS, N.; SECOMB, B.; HULETT, H. How well do you know your clients?: international students' preferences for learning about library services. Australian Academic \& Research Libraries, v. 39, n. 1, p. 38-55, 2008.

SÁNCHEZ-TARRAGÓ, N.; SANTOS, R. N. M.; BUFREM, L. S. Internacionalización de la educación superior: una mirada a sus simientes intelectuales. In: ENCONTRO NACIONAL DE PESQUISA EM CIÊNCIA DA INFORMAÇÃO: ALÉM DAS NUVENS, EXPANDINDO AS FRONTEIRAS DA CIÊNCIA DA INFORMAÇÃO, 15., 2014, Belo Horizonte, MG. Anais... Belo Horizonte: ECI, 2014. p. 3098-3118. Disponível em: $<$ http://enancib2014.eci.ufmg.br/documentos/anais/anais-gt7>. Acesso em: 14 jul. 2016.

SÁNCHEZ-TARRAGÓ, N.; SANTOS, R. N. M.; BUFREM, L. S. Discusión sobre Políticas de Internacionalización en la Educación Superior: análisis de citas. Encontros Bibli: revista eletrônica de biblioteconomia e ciência da informação, v. 20, n. 44, p. 105-126, set./dez. 2015. 
SÁNCHEZ-TARRAGÓ, N.; BUFREM, L. S.; SANTOS, R. N. M. La Ciencia de la Información y la internacionalización de la educación superior. Informação \& Sociedade: estudos, v. 26, n. 1, p. 73-89, 2016.

SAW, G. et al. Social media for international students-it's not all about Facebook. Library Management, v. 34, n. 3, p. 156-174, 2013.

SHAO, X. et al. Chinese students in American academic libraries: a survey of chinese user satisfaction with US library experience. The International Information \& Library Review, v. 45, n. 1-2, p. 28-36, 2013.

SHAO, X.; SCHERLEN, A. Chinese academic libraries serving international students and scholars: an assessment of three case studies. The International Information \& Library Review, v. 43, n. 1, p. 53-61, 2011.

SCHOMBERG, J; GRACE, M. Expanding a collection to reflect diverse user populations. Collection Building, v. 24, n. 4, p. 124-126, 2005.

SIN, S. C. Demographic differences in international students' information source uses and everyday information seeking challenges. The Journal of Academic Librarianship, v. 41, n. 4, p. 466-474, 2015.

SMITH, B. J. The case for international collaboration in academic library management, human resources and staff development. International Federation of Library Associations and Institutions, v. 41, n. 2, p.140-152, 2015.

SOMERVILLE, M. M. et al. At home in the world International library staff exchange program highlights. IFLA journal, v. 41, n. 4, p. 326-335, 2015.

TORRAS, M. C.; VAAGAN, R. Websites and internationalization: a survey of norwegian academic, research and special libraries. Libri, n. 56, p. 28$37,2006$.

UNESCO. Global flow of tertiary-level students. 2015. Disponível em: <http://www.uis.unesco.org/Education/Pages/international-student-flowviz.aspx\#sthash.AWuwcinC.dpuf>. Acesso em: 14 jul. 2016

XU, J; KANG, Q; SONG, Z. The current state of systematic reviews in library and information studies. Library \& Information Science Research, v. 37, n. 4, p. 296-310, 2015.

WITT, S. W.; KUTNER, L.; COOPER, L. Mapping academic library contributions to campus internationalization. College \& Research Libraries, [S.I.], v. 76, n. 5, p. 587-608, July 2015. Disponível em: <http://crl.acrl.org/index.php/crl/article/view/16449>. Acesso em: 10 set. 2017.

YE, Y. New thoughts on library outreach to international students. Reference Services Review, v. 37, n. 1, p.7-9, 2009.

ZHANG, L. Communication in academic libraries: an East Asian perspective, Reference Services Review, v. 34, n. 1, p. 164-176, 2006.

ZIMERMAN, M. Plagiarism and international students in academic libraries. New Library World, v. 113, n. 5-6, p. 290 